\title{
DESENVOLVIMENTO DE HABILIDADES EMPREENDEDORAS PELO USO DA FERRAMENTA EDLE EM DIFERENTES INSTITUIÇÕES E PERÍODOS DE CURSOS DE ENGENHARIAS DO BRASIL
}

Karla Silva - karla@utfpr.edu.br

Universidade Tecnológica Federal do Paraná,

R. Rosalina Maria Ferreira, 1233 - Vila Carola

87301-899 - Campo Mourão - PR

Elzo A. Aranha - eaaranha@ unifei.edu.br

Universidade Federal de Itajubá,

Av. B P S, 1303 - Pinheirinho

37500-903 - Itajubá - MG

Resumo: A nova revolução industrial estabelece aos engenheiros a necessidade de novas competências, implicando na urgência de inovações e evoluções no ensino de engenharia. Neste contexto, a educação empreendedora possibilita desenvolvimento de habilidades típicas dos empreendedores, como liderança, visão, energia, "networking" $e$ "weltanschauung". Em atenção a essas demandas, as mais recentes Diretrizes Curriculares Nacionais (DCN) para engenharias apontam, reiteradamente, para necessidades de um engenheiro com competências e habilidades empreendedoras. Constata-se a ausência de análises acadêmicas sobre o uso da ferramenta EDLE (Entrepreneurial Dynamic Learning) no desenvolvimento de competências empreendedoras dos estudantes, expndo uma lacuna na literatura acadêmica na educação da engenharia. O objetivo da pesquisa é analisar as contribuições da ferramenta EDLE, em instituições educacionais brasileiras, para o desenvolvimento de competências empreendedoras dos estudantes de engenharia. A ferramenta utiliza princípios da educação empreendedora, aprendizagem ativa, design thinking e taxonomia de Bloom. A coleta de dados contou com a participação de 7 professores de diferentes instituições de ensino, envolvendo 257 alunos, do $1^{\circ}$ ao $8^{\circ}$ período, de 13 diferentes cursos de engenharias, além dos cursos de Arquitetura e Química. Os resultados da pesquisa preenchem a lacuna exposta e produzem implicações práticas para coordenadores e professores dos cursos de graduação em engenharia.

Palavras-chave: EDLE. Educação empreendedora. Engenharia. Taxonomia de Bloom.

\section{INTRODUÇÃO}

A nova revolução industrial exige um engenheiro com habilidades alinhadas a um novo cenário de avanços na automação e tecnologia, implicando na urgência de inovações do processo de ensino universitário. Em atenção a essas demandas, as mais recentes Diretrizes Curriculares Nacionais (DCN), para engenharias apontam, reiteradamente, para necessidades de um engenheiro com competências e habilidades empreendedoras. As novas DCNs 
ratificam que as atitudes empreendedoras são necessárias aos novos profissionais das engenharias, na perspectiva de novo paradigma da educação empreendedora (KIRBY, 2007).

Por outro lado, muitos docentes estão buscando pela renovação de suas práxis nas salas de aulas dos cursos de graduação em engenharia (ARANHA, SANTOS e GARCIA, 2018; SUCALA, CARROL e CORY, 2019; TORGEI e LISE, 2020). Uma das iniciativas refere-se a adoção de metodologia para tornarem suas aulas mais dinâmicas, por exemplo, fazendo uso de ferramentas integradas (design thinking, aprendizagem ativa, taxonomia de Bloom, aprendizagem baseada em valor, etc) convenientemente selecionadas e aplicadas (ARANHA, SANTOS e GARCIA, 2018). A ferramenta integrada de aprendizagem é aquela que está fundamentada em duas ou mais abordagens ou princípios, sendo um deles a educação empreendedora, e tem como objetivo o desenvolvimento de competências dos estudantes de engenharia.

A ferramenta EDLE-Entrepreneurial Dynamic Learning (aprendizagem dinâmica empreendedora) está ancorada e conecta a educação empreendedora, aprendizagem ativa, design thinking e taxonomia de Bloom, fomentando o desenvolvimento de competências e habilidades empreendedoras dos estudantes de engenharia (ARANHA et al., 2018). No entanto, os resultados obtidos da pesquisa com a ferramenta EDLE utilizada aos longo de vários anos, estão restritos a uma universidade, conforme destaca Aranha et al. (2018). Logo, torna-se necessário ampliar a utilização dessa ferramenta nos cursos de graduação de engenharia nas diversas instituições educacionais brasileiras, visando analisar o que é capaz de contribuir para desenvolvimento de competências empreendedoras nesses estudantes.

Contudo, a ausência de artigos acadêmicos brasileiros explorando as contribuições da ferramenta EDLE no desenvolvimento de competências empreendedoras dos estudantes, expõe uma lacuna na literatura acadêmica nacional de educação em engenharia. Nesta direção, a questão básica que norteia a presente pesquisa é: Quais são as contribuições da ferramenta EDLE, nas instituições educacionais brasileiras, para o desenvolvimento de competências empreendedoras dos estudantes de engenharia?

O objetivo da pesquisa é analisar as contribuições da ferramenta EDLE, em instituições educacionais do Brasil, para o desenvolvimento de competências empreendedoras dos estudantes de engenharia. A pesquisa é um estudo de caso múltiplo, iniciada em maio de 2019 e concluída em maio de 2020. Como tal, implicou na coleta de dados do total de 257 estudantes de engenharia, de 13 diferentes cursos de Engenharias, e envolveu 07 professores de 07 diferentes instituições de ensino superior.

$\mathrm{O}$ artigo está estruturado em sete seções. Na primeira seção encontra-se a introdução. Na segunda seção estão os principais elementos da revisão da literatura. Na terceira seção encontram-se os métodos e técnicas e na quarta seção estão os resultados. Na quinta seção estão lições aprendidas, na sexta estão as contribuições inovadoras e na última seção as considerações finais.

\section{FUNDAMENTAÇÃO TEÓRICA}

\subsection{A Noção de Educação Empreendedora}

O modelo conceitual da aprendizagem dinâmica empreendedora EDLE, visando fomentar o desenvolvimento de competências do estudante de engenharia, está apoiado em quatro pilares: educação empreendedora, aprendizagem ativa, design thinking e taxomonia de Bloom (ARANHA et. al., 2018). Esta subseção, dedica-se à educação empreendedora e na próxima subseção à aprendizagem ativa, design thinking e taxonomia de Bloom.

A literatura acadêmica apresenta diversas noções e definições de educação empreendedora (GARAVAN e O'CINNEIDE, 1994; GIBB, 2002; FAYOLLE e GAILLY, 
2008; FAYOLLE e VERZAT, 2016; FAYOLLE, 2013; GIBB e PRICE, 2014). A noção ampla de educação empreendedora refere-se à conteúdos, métodos de ensino, aprendizagem e atividades de apoio à criação de conhecimento, desenvolvimento da cultura empreendedora, mentalidade empreendedora, competência, comportamento, atitudes, intenções e valores empreendedores essenciais para o indivíduo viver, trabalhar e criar valor compartilhado na sociedade (GIBB, 2002; FAYOLLE e GAILLY, 2008; FAYOLLE e VERZAT, 2016; FAYOLLE, 2013; GIBB e PRICE, 2014, PORTER e KRAMER, 2011). Na perspectiva da noção de educação empreendedora o estudante de engenharia é o protagonista do processo de aprendizagem, sendo engajado pelo professor facilitador, que adota a postura inovadora em sala de aula.

Fink, Ambrose e Susan (2005) propõem novos papéis do professor de engenharia para uma nova era. Nesses novos papéis, o ciclo da prática profissional do professor inicia com a indentificaçã do problema educacional da sociedade (Fink, Ambrose e Susan, 2005). No entanto, esses autores não destacam a educação empreendedora como um dos papéis essenciais da prática do professor de engenharia. Levando em consideração Fink, Ambrose e Susan (2005) e os referenciais conceituais apresentados anteriormente de educação empreendedora, o quadro 1 estabelece a correspondência entre docência e educação empreendedora. É interessante enfatizar que o professor ao utilizar a ferramenta EDLE adota postura de facilitador e fomentador do processo de aprendizagem.

Quadro 1. Estruturação para verificação de correspondências entre Docência e Educação Empreendedora.

\begin{tabular}{|c|c|}
\hline Doc & orismo \\
\hline $\begin{array}{l}\text { Professor inovador por excelência: } \\
\text { - Caminha com os próprios pés e capaz de investir } \\
\text { capital próprio (se necessário); } \\
\text { - Muitas vezes sozinho, autônomo e dedicado; } \\
\text { - Reestrutura mentalidade, hábito, costume: enfrenta } \\
\text { o medo e parte para batalha! }\end{array}$ & $\begin{array}{l}\text { Empreendedor é uma pessoa que: } \\
\text { - Caminha com os próprios pés e muitas vezes } \\
\text { investe capital próprio; } \\
\text { - Atua frequentemente sozinho, de forma autônoma } \\
\text { e dedicada; } \\
\text { - É reestruturador de mentalidade, hábito, costume: } \\
\text { enfrenta o medo e parte para batalha! }\end{array}$ \\
\hline $\begin{array}{l}\text { Educação Empreendedora: } \\
\text { - É uma "proposta jovem"; } \\
\text { - Ambiente na educação é de incertezas; } \\
\text { - A Educação precisa gerar inovação; } \\
\text { - É repetível, escalável, de baixo } \\
\text { manutenção. }\end{array}$ & $\begin{array}{l}\text { presa: } \\
\text { ovem; } \\
\text { mbiente de incertezas; } \\
\text { a gerar inovação; } \\
\text { o de negócio: repetível, escalável, de } \\
\text { de manutenção. }\end{array}$ \\
\hline
\end{tabular}

Fonte: Elaborado pelos autores

\subsection{Aprendizagem Ativa, Design Thinking e Taxonomia de Bloom}

O segundo pilar da aprendizagem dinâmica empreendedora (EDLE) é a aprendizagem ativa que envolve um conjunto de métodos de aprendizagem que tem como objetivo o engajamento do estudante no processo de aprendizagem (PRINCE, 2004; ARANHA et al., 2018). Dois elementos centrais estão embutidos na aprendizagem ativa, as atividades do estudante e o engajamento no processo de aprendizagem (PRINCE, 2004). Nesta direção, a aprendizagem colaborativa, aprendizagem cooperativa e aprendizagem baseada em problemas são métodos de aprendizagem ativas que encorajam o estudante de engenharia no processo de aprendizagem (PRINCE, 2004; CHRISTERSSON et al., 2019; JOHNSON e JOHNSON, 2018; FREEMAN et al. 2014).).

O terceiro pilar do modelo conceitual da aprendizagem dinâmica empreendedora (EDLE) é o design thinking que estabelece cinco espaços de inovação para o desenvolvimento de projetos (BROWN, 2008; ARANHA et al., 2018). Por meio do desenvolvimento de protótipos ao longo dos cincos estágios de inovação, o foco do design thinking é o 
desenvolvimento de soluções inovadoras centrado no usuário, ou seja, no interessado da solução. O quarto pilar refere-se à taxonomia de Bloom. É uma abordagem que amplia a compreensão dos objetivos da aprendizagem e os domínios cognitivos que o professor pretende desenvolver no estudante (BLOOM, 1956; ARANHA et al., 2018). Os domínios cognitivos estão agrupados em atividades do pensamento da mais baixa ordem e atividades do pensamento da mais alta ordem.

Os processos utilizados, em cada etapa, com as respectivas metodologias de aprendizagem, domínios cognitivos esperados pelos professores e espaços de inovação seguiram o procedimento descrito por ARANHA et al. (2018).

\section{MÉTODOS E TÉCNICAS}

A pesquisa é exploratória. A partir da identificação da lacuna existente, na literatura acadêmica de educação em engenharia, foi estabelecida a formulação do problema que orientou o desenvolvimento da pesquisa (LAKATOS; MARCONI. 2003). A pesquisa adota o estudo de caso múltiplo envolvendo 06 disciplinas de 07 instituições de educação superior, federal e particular brasileiras (YIN, 2005). A pesquisa foi desenvolvida em quatro etapas. $\mathrm{Na}$ primeira etapa foi realizada a revisão da literatura. A segunda etapa constituiu em um curso de capacitação introdutório de 8 horas sobre a ferramenta EDLE para mais de 40 professores envolvidos. Voluntariamente 07 professores de 07 diferentes instituições de ensino superior pública e privada introduziram a ferramenta EDLE nas disciplinas ministradas. Esses docentes, devidamente treinados, em um curso realizado em maio de 2019, elegeram as disciplinas que ministravam e onde utilizaram o EDLE: Metodologia Científica e Metodológica, Metodologia de Projeto e Produto, Instrumentação e Controle de Processos, Introdução à Engenharia, Introdução ao Empreendedorismo, Termodinâmica Aplicada.

As disciplinas selecionadas englobaram alunos de diferentes períodos (variando do $1^{\circ}$ ao $8^{\circ}$ ), e os professores seguiram o cumprimento dos roteiros de implementação da ferramenta que foram enviados semanalmente. Participaram desse projeto um total de 257, de 13 diferentes cursos de Engenharias: Aeroespacial, de Alimentos, Bioprocessos e Biotecnologia, de Controle e Automação, de Materiais, de Transporte e Logística, Aeromotiva, Civil, Elétrica, Ferroviária e Metroferroviária, Mecânica, de Produção e Química; além de alunos dos cursos de Arquitetura e de Licenciatura em Química. A terceira etapa foi a coleta de dados que consistiu no preenchimento do questionário Google Form pelos alunos no final da disciplina. A quarta etapa foi a análise de dados e dos resultados.

\section{RESULTADOS E DISCUSSÃO}

\subsection{A ferramenta EDLE em sala de aula}

O Quadro 2 contêm as 12 etapas sequenciais, desenvolvidas ao longo de 3 meses pelos professores envolvidos no projeto, segundo critérios de tempo utilizado e condições de operacionalização estabelecidos por cada um dos professores que investiram seus tempos e energia na ação.

A primeira ação, Etapa 1 da metodologia, foi o lançamento do desafio ao aluno, isto é, cada aluno precisou propor 3 ideias (3 problemas e uma solução para cada um desses problemas propostos). As etapas subsequentes evoluiram para avaliação, culminando na seleção da melhor ideia. Pode-se observar (Quadro 2) que a ação previa um início individual do aluno, que apresentaria suas ideias a serem analisadas e avaliadas pelos pares, culminando 




"Os desafios para formar hoje o engenheiro do amanhã"

na formação de equipes para desenvolvimento das etapas de prototipação e modelos de negócio para as melhores ideias (segundo avaliações dos participantes).

Objetivando investigar a percepção do aluno em relação ao nível cognitivo trabalhado em relação a Taxonomia de Bloom, os professores fizeram um fechamento instruindo seus alunos a responderem anonimamente, um questionário estruturado.

Quadro 2. Etapas de operacionalização da ferramenta "EDLE"

\begin{tabular}{|c|c|c|}
\hline Etapa & Descrição da etapa & Realização \\
\hline 1 & 3 Ideias inovadoras & Individual \\
\hline 2 & Seleção da Ideia Principal & Individual \\
\hline 3 & Apresentação Oral de uma das três ideias & Individual \\
\hline 4 & Escolha por Votação das Ideias Principais & Individual \\
\hline 5 & Apresentação Objetivos de Ideias mais Votadas & Equipe \\
\hline 6 & Detalhamento nas Ideias nos Grupos & Equipe \\
\hline 7 & Apresentação da Ideia Detalhada + Instrumento de Questionário \\
\hline 8 & Revisão da Solução/Questionário & Equipe \\
\hline 9 & Aplicação do Questionário & Equipe \\
\hline 10 & Tabulação dos Resultados & Equipe \\
\hline 11 & Análise dos Resultados/Validação de Mercado da Solução & Equipe \\
\hline 12 & Desenvolvimento Modelo de Negócios + Prototipação & Equipe \\
\hline
\end{tabular}

Fonte: Elaborado pelos autores

No questionário, os alunos inicialmente deveriam selecionar, para cada uma das 12 Etapas, um dentre os seis domínios cognitivos da taxonomia de Bloom (lembrar, entender, aplicar, analisar, avaliar, criar) que teve o desenvolvimento mais significativo sob a percepção do mesmo. Os acadêmicos selecionaram 3 dentre as 5 habilidades segundo metamodelo empreendedor de Filion ("weltanschauung", visão, liderança, energia, "networking") que julgavam ter sido prioritariamente adquiridas com o desenvolvimento EDLE.

Os professores participantes do projeto, foram convidados ao fórum virtual, onde puderam expressar suas percepções, experiências e tecer considerações importantes ao tema.

\subsection{Análise de dados e resultados}

A percepção dos acadêmicos quanto as habilidades adquiridas, segundo metamodelo empreendedor de Filion (1993), encontram-se reportadas na Figura 1. Constata-se que a maioria dos alunos $(80 \%)$, concordaram que a atuação no desenvolvimento do projeto EDLE, conferiu visão, seguidos pelos quase $60 \%$ que admitiram terem adquirido habilidades no estabelecimento de relações (networking) e liderança.

Figura 1. Percepção de 112 acadêmicos, das habilidades

112 respostas

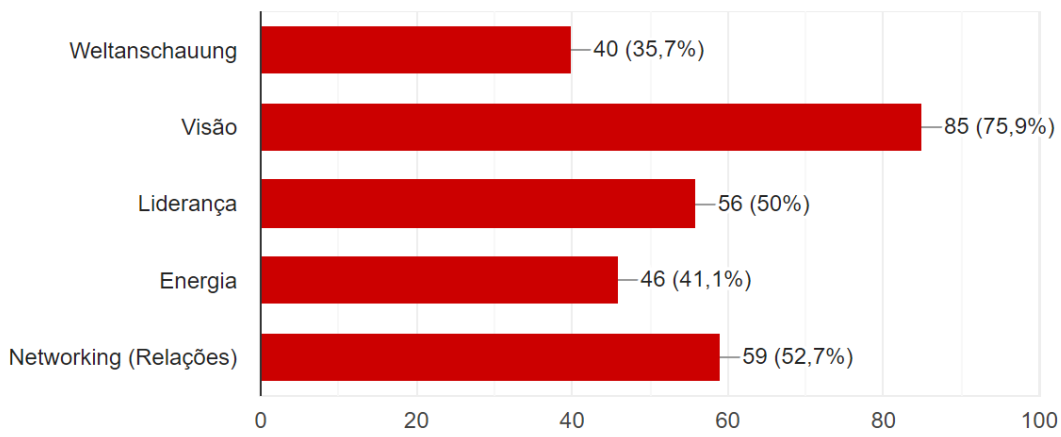


Conforme expostos no Quadro 3, 112 dos 257 acadêmicos participantes do projeto responderam aos questionamentos (44\%) de percepções pessoais quanto aos níveis cognitivos segundo a Taxonomia de Bloom, para cada uma das etapas EDLE.

Quadro 3. Percepção de 112 acadêmicos, que retornaram respostas, quanto aos níveis cognitivos, segundo Taxonomia de Bloom, para cada uma das Etapas do processo EDLE.

Etapa 1: Desenvolvimento de 3 Etapa 2: Escolha 1 ideia a ser Etapa 3: Apresentação oral ideias (produto, serviço, tecnologia, apresentada aos colegas - ideia processo) analisando possibilidades. principal.

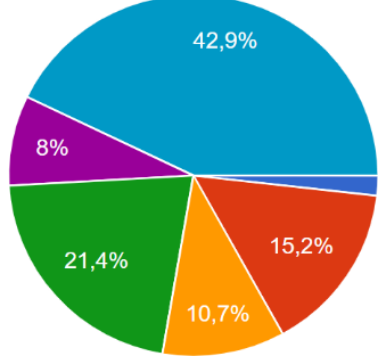

Etapa 4: Votação para escolha das 10 melhores ideias principais a serem desenvolvidas em equipes.

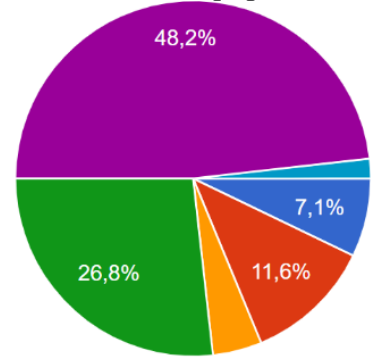

Etapa 7: Apresentação do protótipo, com instrumentos de validação financeira/ mercado, para feedback.

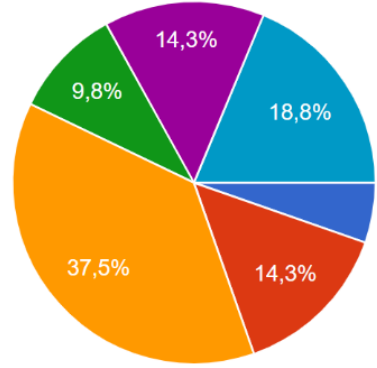

Etapa 10: Tabulação de dados da validação financeira e de mercado

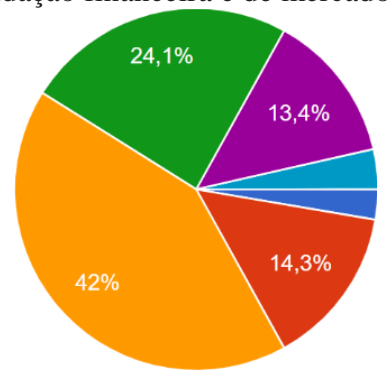

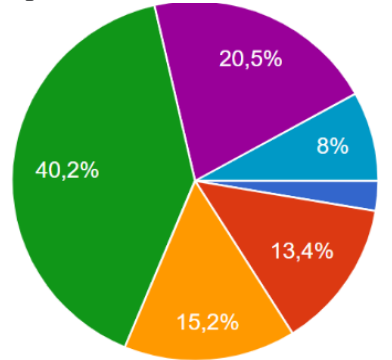

Etapa 5: Apresentação individual (3 min.) de melhor idéia escolhida, para expor visão de futuro.

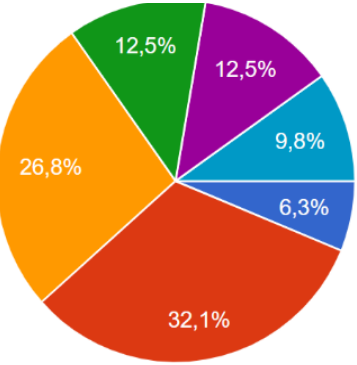

Etapa 8: Brainstorming, revisão/ complemento do protótipo. Instrumentos de validação financeira/ mercado.

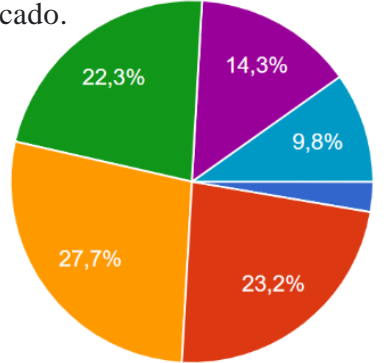

Etapa 11: Análise de dados

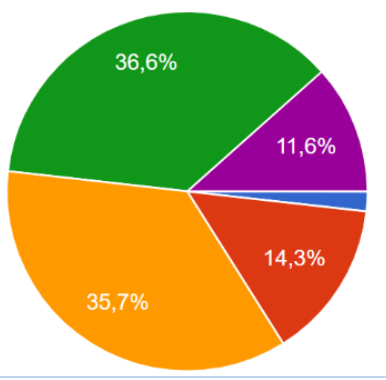

individual de 1 minuto, em sala de aula, sobre a ideia principal.

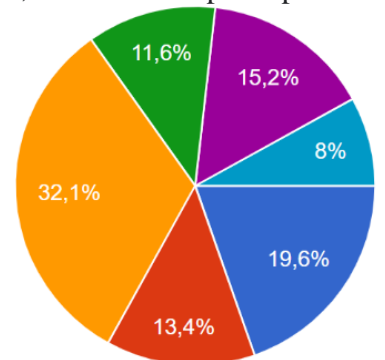

Etapa 6: Formação de Equipes para o desenvolvimento das melhores ideias principais escolhidas.

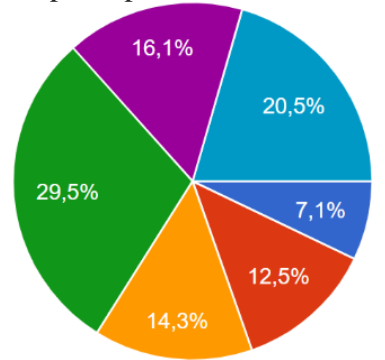

Etapa 9: Validação da ideia principal externa pelas equipes (entrevistas com 100 pessoas ou 30 empresas).

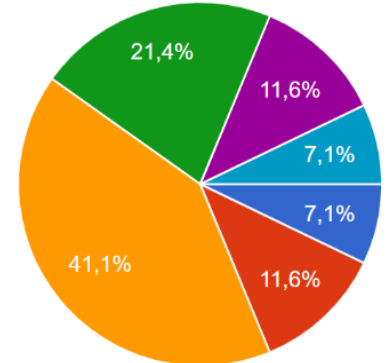

Etapa 12: Prototipagem final e desenvolvimento da modelagem de negócios.

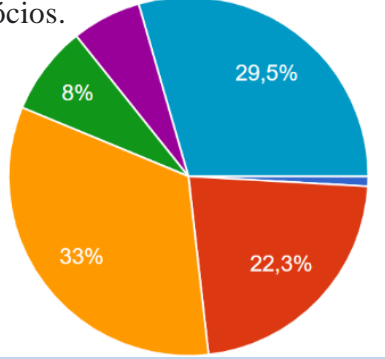

Legenda:

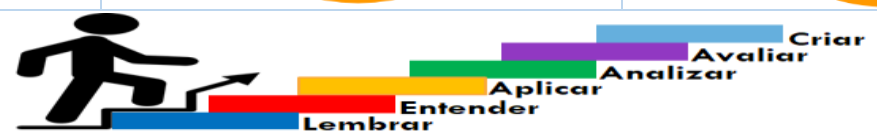


Os resultados apontam que a metodologia utilizada foi capaz de mobilizar os alunos para diferentes níveis congnitivos, tornando ainda possível o trabalho com níveis cognitivos de ordem superior, tais quais nos processos de análise, avaliação e criação.

Quanto aos apontamentos feitos pelos professores promotores do uso da ferramenta EDLE neste projeto, todos concordaram que ela possibilitou uma dinâmica mais estruturada, promovendo maior envolvimento dos acadêmicos e com resultados bastante animadores. Todos os professores produziram trabalhos científicos específicos com detalhes de seus resultados e divulgação de eventuais produtos oriundos dessa iniciativa.

Um resumo das percepções, prospecções e considerações desses professores, será estabelecido nos dois próximos itens, na forma de lições aprendidas, contribuições inovadores e implicações práticas.

\section{LIÇÕES APRENDIDAS}

Embora existam muitas metodologias ativas, é importante que a escolha ou uso das mesmas estejam alinhados com a construção dos saberes e competências, numa ótica progressiva do desenvolvimento cognitivo. A ferramenta EDLE tornou possível essa integração de ferramentas, possibilitando ainda um maior envolvimento e prazer do aluno em participar do processo, apresentando resultados concretos. Para sucesso do procedimento pedagógico adotado pelo professor, como observado pelos professores que trabalharam com o EDLE, é fundamental que se identifique a disciplina/ unidade curricular, bem como que se tenha consciência do caminho e avalie os resultados para garantia do desenvolvimento almejado de elementos de competência. Todo esse processo, portanto, exige um planejamento sólido e o professor deve estar atento às necessidades de seus "clientes" (alunos), promovendo uma problematização que considere mercado de trabalho e contextos sociais. O processo exige que o professor seja intrépido, e que reconheça e, para maior eficácia ao curso, sempre que possível, alie-se a professores de diversas áreas, promovendo a interdisciplinaridade, multidisciplinaridade e transdiciplinaridade. Ainda, é fundamental que sejam verificados os indicadores, sempre na busca de que os resultados de aprendizagem sejam atingidos.

\section{CONTRIBUIÇÕES INOVADORAS E IMPLICAÇÕES PRÁTICAS}

Os resultados gerados pela presente pesquisa proporcionam contribuições inovadoras uma vez que os resultados preenchem a lacuna exposta no campo da educação em engenharia. A lacuna exposta trata da ausência de artigos acadêmicos brasileiros, analisando as contribuições da ferramenta EDLE, nas instituições educacionais brasileiras, no desenvolvimento de competências empreendedoras dos estudantes de engenharia.

Quanto aos implicadores práticos destacam-se: 1) O EDLE é uma ferramenta capaz de desenvolver, em alunos de engenharia, competências empreendedoras; 2) Diretores e coordenadores de cursos de engenharias poderão desenvolver palestra de sensibilização, para professores, promovendo a Educação Empreendedora; 3) Professores e coordenadores poderão inserir no PPC uma ferramenta que faz uso integrado de diferentes metodologias ativas, promovendo ainda trabalho conjunto entre as várias áreas das Engenharias; 4) Instituição, como um todo pode criar e estimular, implantação de ferramentas, plataformas, ecossistemas, no processo de geração e validação de ideias; 5) Potencializar na capacidade de gerar soluções inovadoras para sociedade, aumentando o impacto da Universidade. 


\section{CONSIDERAÇÕES FINAIS}

A pesquisa envolveu a coleta de dados do total de 257 estudantes de engenharia, de 13 diferentes cursos de Engenharias, envolvendo 07 professores de 07 diferentes instituições de ensino superior de maio a dezembro de 2019. A amostra é representativa para a nossa investigação. Os resultados obtidos oferecem contribuições inovadores e produzem implicações práticas. Logo, o objetivo da pesquisa foi plenamente atingido.

$\mathrm{O}$ presente trabalho desenvolveu algumas inferências entre docência $\mathrm{e}$ empreendedorismo, torna-se conveniente concluir nos aproximando novamente da terminologia clássica utilizada no mundo do emprendedorismo. Assim, finaliza apontando aspectos do "mindset" ideal para colocar em prática o "lean startup" (aqui representado pelo EDLE), em que sugere aos professores que pretendem ressignificar a práxis docente para formação do novo engenheiro: teste, erre e corrija rápido: falhar é totalmente aceitável, desde que seja gastando poucos tempo e dinheiro; o aprendizado é o sucesso do projeto: se aproxima da solução ideal entregando mais valor à educação; decisões precisam estar alinhadas com as perspectivas do cliente (interno ou externo); aprender fazendo: discutir menos e testar mais; manter mente de iniciante $=$ abertos a testar e descobrir coisas novas; ser orientado por dados: informações como orientadores nas tomadas de decisão; celebrar o sucesso: toda mudança de mentalidade é desafiadora, logo, teste que foi colocado em prática é motivo de comemoração e o teste que não foi bem sucedido em termos de indicadores, mas gerou ricos aprendizados, também deve ser celebrado.

\section{Agradecimentos}

Agradecemos aos professores e pesquisadores parceiros que se disponibilizaram a participar tão ativamente desta pesquisa, exímios professores e/ou pesquisadores das instituições: FAAP, ENCE-IBGE, UniCBE, UFSC, UNIFEI, UTFPR-CM, UTFPR-PG.

\section{REFERÊNCIAS}

AADLAND, T.; AABOEN, L. An entrepreneurship education taxonomy based on authenticity, European Journal of Engineering Education, 2020. Disponível em: https://ntnuopen.ntnu.no/ntnuxmlui/bitstream/handle/11250/2645069/AadlandAaboenTaxonomy.pdf?sequence=1. Acesso em 20 jul 2020.

ARANHA, E.; DOS SANTOS, P.; GARCIA, N. P. EDLE: An integrated tool foster entrepreneurial skills development in engineering education. Educational Techonology Research and Development, 2018.

BLOOM B. S. Taxonomy of Educational Objectives: The Classification of Educational Goals. p. 201-207; Editora B. S. Bloom. David McKay Company, Inc. 1956. Acesso em: 09 fev. 2020.

BROWN, T. Design Thinking. Harvard Business Review, 85-92, 2008. Disponível em: https://hbr.org/2008/06/design-thinking. Acesso em: 20 jul. 2020.

CHRISTERSSON, Cecilia et al. Promoting Active Learning in universities- Thematic Peer Group Report. European University Association, 2019. Disponível em: 
(C) COBENCE

"Os desafios para formar hoje o engenheiro do amanhã"

https://eua.eu/resources/publications/814:promoting-active-learning-in-universities-thematicpeer-group-report.html. Acesso em: 01 jul. 2020.

FAYOLLE, A.; BENOIT, G. From craft to science: Teaching models and learning processes in entrepreneurship education. Journal of European Industrial Training, v. 32, n. 7, p.569$593,2008$.

FAYOLLE, A.; VERZAT, C. In quest of legitimacy: The theoretical and methodological foundations of entrepreneurship education research. International Small Business Journal, v. 34, n.7, p. 895-904, 2016.

FAYOLLE, A. Personal views on the future of entrepreneurship education,

Entrepreneurship and Regional Development, v. 25, n. 7-8, p. 692-701, 2013.

FILION, L. J. Visão e Relações: elementos para um metamodelo empreendedor. Revista de Administração de Empresas. São Paulo. p. 50-61, 1993

FINK, L.; AMBROSE, S.; WHEELER, D. Becoming a professional engineering educator: A new role for a new era. Journal of Engineering Education. v. 94, n. 1, p. 185-194, 2005.

FREEMAN, S. et al. Active learning increases student performance in science, engineering, and mathematics. National Academy of Sciences of the United States of America, v. 111, n. 23, p. 8410-8415, 2014.

GARAVAN, T. N.; O'CINNEIDE, B. Entrepreneurship Education and Training Programmes: A Review and Evaluation - Part 1. Journal of European Industrial Training, v. 18, n. 8, p. 3-12, 1994. Disponível em: https://doi.org/10.1108/03090599410068024. Acesso em 25 jul. 2020 .

GIBB, A. Pursuit of a New "Enterprise" and "Entrepreneurship" Paradigm for Learning: Creative Destruction, New Values, New Ways of Doing Things and New Combinations of knowledge. International Journal of Management Reviews, v. 4, p. 213-231, 2002.

GIBB, A.; PRICE, A. A Compendium of Pedagogies for Teaching Entrepreneurship. 2nd Edition, 2014.

JOHNSON, D. W.; JOHNSON, R. Cooperative Learning: The Foundatioin for Active Learning-Provisional Chapter. IntechOpen, 2018.

KIRBY, D. Changing the entrepreneurship education paradigm. In: Handbook of Research in Entrepreneurship Education, v. 1: A general perspective, edited by Alain Fayole, Edgard Elgar, 2007.

LAKATOS, E. M.; MARCONI, M. A. Fundamentos de metodologia científica. 5. ed. São Paulo: Atlas, 2003.

NABI, G. et al. The impact of Entrepreneurship Education in Higher Education: A systematic Review and Research Agenda. Academy of Management Learning \& Education, v. 15, n. 2, 2017. 
PRINCE, M. Does Active Learning Work? A Review of the Research. Journal of Engineering Education, Jul. 2004.

PORTER, M. E.; KRAMER, M. R. Creating share value. Harvard Business Review, v. 89, n. 1-2, p. 1-17, 2011.

SHANE, S. VENKATARAMAN, S. The promise of entrepreneurship as a field of research. Academy of Management Review, v.25, n. 1, 2000.

SUCALA, V.I; CARROL, S.; CORY, C.Z. A new model of entrepreneurship education for engineering students. Proceedings of the SEFI 47th Annual Conference Concept Paper, Budapest, p. 16-20, set. 2019

YIN, R.K. Estudo de caso: Planejamento e Método. Porto Alegre: Bookman, 2005.

\title{
DEVELOPING ENTREPRENEURIAL SKILLS BY THE USE OF THE EDLE TOOL IN DIFFERENT INSTITUTIONS AND ENGINEERING COURSE PERIODS IN BRAZIL
}

\begin{abstract}
The new industrial revolution establishes key issues for new engineers' skills, taking the urgency of innovations and evolutions in engineering education. In this context, entrepreneurship education enables the development of typical skills of entrepreneurs, such as leadership, vision, energy, "networking" and "weltanschauung". In response to these key issues, the most recent National Curricular Guidelines (DCN) for engineering repeatedly point to an engineer with entrepreneurial skills and abilities. There is a lack of academic analysis on the use of the EDLE tool (Entrepreneurial Dynamic Learning) in the development of students' entrepreneurial skills, exposing a gap in the academic literature in engineering education. The objective of the research is to analyze the contributions of the EDLE tool, in Brazilian educational institutions, to the development of entrepreneurial skills of engineering students. The tool uses principles of entrepreneurship education, active learning, design thinking and Bloom's taxonomy. The data collection was attended by 7 professors from different educational institutions, involving 257 students, from the 1st to the 8th period, from 13 different engineering courses, in addition to the Architecture and Chemistry courses. The research findings fill the gap and have several practical implications for coordinators and professors of undergraduate engineering courses.
\end{abstract}

Keywords: EDLE. Entrepreneurial Education. Engineering. Bloom's taxonomy. 